\title{
Editorial
}

\section{Synergies between the Convention on Biological Diversity (CBD) and the United Nations Framework Convention on Climate Change (UNFCCC).}

As a decision of the $9^{\text {th }}$ Conference of Parties of CBD an Ad Hoc Technical Expert Group (AHTEG) was established to provide biodiversity related information to (UNFCCC), through the provision of scientific and technical advice and assessment on the integration of the conservation and sustainable use of biodiversity into climate change mitigation and adaptation activities.

Listed below are some of the major points raised by the first report of this AHTEG, and the full document is available at http://www.cbd.int/doc/meetings/cc/ahteg-bdcc-01/other/ahteg-bdcc-01-findings-en.pdf.

Maintaining natural and restoring degraded ecosystems, and limiting human-induced climate change, represent multiple benefits for both the (UNFCCC) and CBD if mechanisms to do so are designed and managed appropriately. Well-functioning ecosystems are necessary to meet the objective of the (UNFCCC) owing to their role in the global carbon cycle, their significant carbon stocks and their contribution to adaptation. Carbon is stored and sequestered by biological and biophysical processes in ecosystems, which are underpinned by biodiversity. An estimated 2,400 Gt C is stored in terrestrial ecosystems, compared to approximately $750 \mathrm{Gt}$ in the atmosphere. Carbon stored in soil accounts for a high percentage of the carbon stored in terrestrial ecosystems. Furthermore, well-functioning ecosystems have greater resilience to climate change which will aid in their natural adaptation, and contribute to the assurance of long-term sustainable development under changing climatic conditions.

Maintaining and restoring ecosystems represents an opportunity for win-win benefits for carbon sequestration and storage, and biodiversity conservation and sustainable use. Co-benefits are most likely to be achieved in situations where integrated and holistic approaches to biodiversity loss and climate change are implemented. Many activities that are undertaken with the primary aim of meeting the objectives of the CBD have significant potential to contribute to the mitigation of climate change. Likewise, many activities that are undertaken or being considered with the primary purpose of mitigating climate change could have significant impacts on biodiversity. In some cases these impacts are negative, and there are trade-offs to be considered.

While protected areas are primarily designated for the purpose of biodiversity conservation, they have significant additional value in storing and sequestering carbon. There are now more than 100,000 protected sites worldwide covering about 12 per cent of the Earth's land surface. A total of $312 \mathrm{Gt}$ carbon or $15.2 \%$ of the global carbon stock is currently under some degree of protection. The designation and effective management of new protected areas, and strengthening the management of the current protected area network, could contribute significantly to climate change mitigation efforts. Given that forests contain almost half of all terrestrial carbon, preliminary studies show that continued deforestation at current rates would hamper significantly mitigation efforts. In fact, if all tropical forests were completely deforested over the next 100 years, it would add about $400 \mathrm{GtC}$ to the atmosphere, and increase the atmospheric concentration of carbon dioxide by about $100 \mathrm{ppm}$, contributing to an increase in global mean surface temperatures of about $0.6{ }^{\circ} \mathrm{C}$.

Emission reductions will not be implemented within a time-frame sufficient to allow ecosystems to adapt naturally. Land use, land-use change and forestry activities, including reduced deforestation and degradation can, in concert with stringent reductions in fossil fuel emissions of greenhouse gases, limit climate change. Reducing emissions from deforestation and forest degradation in developing countries (REDD) in areas of high carbon stocks and high biodiversity values can promote co-benefits for climate change mitigation and biodiversity conservation and sustainable use. The national gap analyses carried out by Parties under the Program of Work on Protected Areas of the CBD can be a valuable tool for identifying areas for the implementation of (REDD) schemes. In order to avoid conflict between the implementation of the CBD and the (UNFCCC), biodiversity considerations could be taken into account in the development of the (REDD) methodology. Standards, indicative guidelines and criteria taking into account biodiversity conservation could be developed to potentially enhance positive benefits on biodiversity.

Anthropogenic changes in climate and atmospheric $\mathrm{CO}_{2}$ are already having observable impacts on ecosystems and species; some species and ecosystems are demonstrating apparent capacity for natural adaptation, but others are showing negative impacts. Impacts are widespread even with the modest level of change observed thus far in comparison to some future projections. Observed signs of natural adaptation and negative impacts include: geographic distributions of species; timing of life cycles 
(phenology); interactions between species due to mismatches between the peak of resource demands by reproducing animals and the peak of resource availability; photosynthetic rates, carbon uptake and productivity in response to $\mathrm{CO}_{2}$ "fertilization" and nitrogen deposition; community and ecosystem structural and functional changes.

The negative impacts of climate change on biodiversity have significant economic and ecological costs, such as: the values and services an ecosystem provides; changes in the distributional pattern of species, including human disease vectors and exposure; changes in water flow regulation; change in agricultural productivity; changes and shifts in the distribution of marine biodiversity with serious implications for fisheries; shifts in phenology and geographic ranges of species could impact the cultural and religious lives of some indigenous peoples; novel environments and novel ecosystems are likely to emerge with potentially unexpected behavior.

There is considerable confidence that climate models provide credible quantitative estimates of future climate change, particularly at continental scales, and they are unanimous in their prediction of substantial warming under greenhouse gas increases. However, at finer spatial scales projections have a high level of uncertainty, particularly in tropical and subtropical regions, and in relation to projections of rainfall change. Available models contain inadequate representations of the interactive coupling between ecosystems and the climate system and of the multiple interacting drivers of global change.

On the other hand, despite efforts like the Global Biodiversity Information Facility/GBIF), there is still a lack of extensive, readily available quantitative information on many species globally, especially at understanding where species are not (a critical factor in performing many bioclimatic models) present. There is uncertainty with respect to the functional role of individual species and the functioning of complex systems. Further uncertainties are drawn from: the assumption of instantaneous (and often perfect) migration, which biases impact estimates; the net result of changing disturbance regimes (especially through fire, insects and land-use change) on biotic feedbacks to the atmosphere, ecosystem structure, function and biodiversity; the magnitude of the $\mathrm{CO}_{2}$-fertilisation effect in the terrestrial biosphere and its components over time; the limitations of climate envelope models used to project responses of individual species to climate changes, and for deriving estimations of species extinction risks (see below); the synergistic role of invasive alien species in both biodiversity and ecosystem functioning; the effect of increasing surface ocean $\mathrm{CO}_{2}$ and declining $\mathrm{pH}$ on marine productivity, biodiversity, biogeochemistry and ecosystem functioning; and the impacts of interactions between climate change and changes in human use and management of ecosystems as well as other drivers of global environmental change in ecosystems including more realistic estimates of lagged and threshold responses.

Despite their limitations, the use of bioclimatic modeling techniques allows a useful, and often accurate first cut assessment of spatial distribution of exposure and vulnerability in relation to conservation efforts. Where expert knowledge on species demography is available, techniques can be applied that include consideration of climate variability and require species abundance data, but this places a higher demand on the spatial and temporal resolution of future climate data. While the use of spatially downscaled future scenario data is ideal if achievable, for robust risk and impacts assessments it may be more useful to focus on a range of future climate scenarios even if they are not downscaled, and not only on a mean or median future scenario

Although regretting that it took more then 15 years to have the obvious synergies between both conventions being officially recognized and evaluated, let's hope that until October 2010, when the $10^{\text {th }}$ CBD COP will take place in Nagoya/Japan, a common agenda has been set.

\section{Carlos Alfredo Joly}

Department of Plant Biology, Biology Institute, State University of Campinas, CP 6109, CEP 13083-970, Campinas/SP, Brazil and Chairman of the BIOTA/FAPESP Program. 